\title{
EVALUACIÓN DE DESEMPEÑO Y ALINEAMIENTO ESTRATÉGICO: BASES PARA UNA FORMULACIÓN MÉTRICA
}

\author{
https://dx.doi.org/10.48097/2674-8673.2021n5p10
}

Julio Miranda Vidal ${ }^{1}$

\section{RESUMEN}

Los retos del desarrollo exigen que todos los actores de una nación converjan hacia un propósito común: un ideario nacional; en este intervienen tres actores fundamentales: El gobierno, la sociedad civil y las empresas. A los gobernantes les cabe la misión de formular las políticas públicas necesarias, a fin de generar las adecuadas condiciones para el logro de los objetivos y metas del ideario, el cual debe ser una monolítica expresión del soberano. A la sociedad civil, desde la individualidad, y desde sus organizaciones, participar activamente de las tareas que impone tamaño desafío. Las empresas, en su rol de producir y servir, deben alinear las estrategias al capital humano, de modo que el logro individual agregado cree valor organizacional sinergizado, esto impone exigentes tareas, desde adecuar los cuadros organizacionales, definir el contenido de los puestos y descriptores de cargo, todo en función de horizontes de largo plazo. Para monitorear el grado de cumplimiento de las expectativas del cargo con los resultados reales es necesario avanzar, con urgencia, hacia modelos de evaluación de desempeño de mayor objetividad y racionalidad que primen por sobre evaluaciones con importante grado de subjetividad.

Palabras clave: Alineamiento estratégico. Evaluación de desenpeño. Formulación métrica. Modelos de evaluación.

Data de submissão: 22/06/2021

Data de aprovação: 28/06/2021

\section{AVALIAÇÃO DE DESEMPENHO E ALINHAMENTO ESTRATÉGICO: BASES PARA UMA FORMULAÇÃO MÉTRICA}

\section{RESUMO}

Os desafios do desenvolvimento exigem que todos os atores de uma nação convirjam para um propósito comum: uma ideologia nacional. Três atores fundamentais intervêm nisso: o governo, a sociedade civil e as empresas. Os governantes têm a missão de formular as políticas públicas necessárias, a fim de gerar as condições adequadas para o cumprimento dos objetivos e metas da ideologia, que deve ser uma expressão monolítica soberana. A sociedade

\footnotetext{
${ }^{1}$ Universidad Mayor, Chile. Profesor de Liderazgo, Innovación, Administración y Gestión de Recursos Humanos, y Coordinador Línea Administración y Recursos Humanos de la Facultad de Emprendimiento y Negocios. Miembro del Panel de Expertos de ONU-PNUD-RedUnirse en Responsabilidad Social. E-mail: mirandavidal@gmail.com
} 
civil, desde a individualidade e de suas organizações, participa ativamente das tarefas que tal desafio impõe. As empresas, no seu papel de produzir e servir, devem alinhar estratégias com o capital humano, de forma que a conquista individual agregada crie valor organizacional sinérgico, isso impõe tarefas exigentes, desde adequar os organogramas, definir o conteúdo dos cargos e descritores cobrados, tudo com base em horizontes de longo prazo. Para acompanhar o grau de cumprimento das expectativas do cargo com os resultados reais é necessário avançar, com urgência, para modelos de avaliação de desempenho de maior objetividade e racionalidade que prevalecem sobre avaliações com significativo grau de subjetividade.

Palavras-chave: Alinhamento estratégico. Avaliação de desempenho. Formulação métrica. Modelos de avaliação.

\begin{abstract}
Development challenges demand that all actors in a nation converge towards a common purpose: a national ideology. Three actors are essential for this: the government, civil society and companies. Governments have the mission of formulating the necessary public policies in order to generate the adequate conditions for the fulfillment of the objectives and goals of the ideology, which must be a sovereign monolithic expression. Civil society, either individually or through its organizations, actively participates in the tasks that such a challenge imposes. Companies, in their role of producing and serving, must align strategies with human capital, so that the aggregated individual achievement creates synergistic organizational value. This imposes demanding tasks, from adapting the organization charts to defining the content of the positions and descriptors charged, all based on long-term horizons. In order to monitor the degree of fulfillment of the job's expectations with the actual results, it is urgently necessary to move towards performance evaluation models with greater objectivity and rationality that prevail over evaluations with a significant degree of subjectivity.
\end{abstract}

Keywords: Strategic alignment. Performance evaluation. Metric formulation. Assessment models.

\title{
INTRODUCCIÓN
}

El siglo XX puede entenderse como un "siglo bisagra" entre el período concluido con el fenómeno de la Revolución Industrial británica y la irrupción de las tecnologías de la información y las comunicaciones.

En este contexto, la gestión organizacional en todos sus ámbitos: público, privado y en entidades de la sociedad civil y de la educación, ha experimentado profundas transformaciones. Los conceptos de Dirección y Gerencia en los albores del siglo pasado son profundamente distintos a los que imperan hoy en las organizaciones.

Esa centuria fue capaz de producir grandes obras del genio humano, también, al menos en el mundo occidental, ha habido dos grandes guerras mundiales y otros tantos conflictos 
localizados, de diverso grado de conmoción. En el período se han usado, consumido y expoliado grandes masas de recursos naturales, muchos de ellos no renovables. Como consecuencia ha emergido con fuerza la disciplina de la Responsabilidad Social, la que ha madurado velozmente desde la filantropía y la cultura verde hasta un replanteamiento del orden civilizatorio, en íntima amalgama con la sustentabilidad de las actividades humanas presentes y futuras.

Es así como la Responsabilidad Social se ha planteado ambiciosos propósitos, tal como lo refleja la Declaración de Panamá de 2012, producto de la Cumbre de expertos patrocinada por el Programa de Desarrollo de las Naciones Unidas: "Nos comprometemos a trabajar en el cultivo, investigación, enseñanza, divulgación y aplicación de los principios de la Responsabilidad Social, para contribuir al desarrollo humano sustentable, en plena armonía con nuestros pueblos y el ambiente.” (DE LA CORTE Y MIRANDA, 2012).

De este modo, los desafíos del management pasan necesariamente por el colosal reto de la Creación de Valor en plena armonía con las ciudadanías y el ambiente. Esto obliga a precisar que el crear valor trasciende objetivos basales clásicos: en las empresas, generar excedentes y solidez patrimonial; en el ámbito público, externalidades positivas; en la sociedad civil, reivindicaciones colectivas. En todos estos casos la sustentabilidad de las estructuras organizacionales es hoy un pilar insoslayable, es decir, la preocupación e incidencia en el bien común proyectado en un horizonte de largo plazo y de carácter global.

Toda organización sin considerar el equipo humano es una simple concurrencia y coexistencia de diversos activos inertes, que no pueden movilizarse, producir ni crear sin la intervención del genio y talento humanos.

Estas reflexiones nos llevan a un punto central en el debate contemporáneo y en la cosmovisión organizacional, la relevancia indesmentible del accionar humano en la dinámica social. Esto pone a este genuino "patrimonio humano" en simetría con el patrimonio económico-financiero de la entidad. Es decir, el valor creado es función de ambos conglomerados, y sus beneficiarios son la empresa, como colectivo humano, los clientes o usuarios, proveedores y la comunidad y el ambiente.

"Crear valor" puede entenderse con meridiana claridad desde lo intelectual, sin embargo, crear valor en el mundo real exige energía, esfuerzos y competencias complejas. De no ser así no habría empresas que fracasan, proyectos frustrados o sociedades inestables, e incluso estados fallidos.

En la actualidad se manejan variadas expresiones y denominaciones para referirse a "las personas" desde la óptica de la organización: gestión de recursos humanos, gestión de 
personas, gestión del talento, administración de recursos humanos, incluso unas tan antiguas como administración de personal. Para nuestros efectos, y entendido el contexto, usaremos a lo largo del texto la expresión "gestión de personas", advirtiendo que no es nuestro objeto expreso profundizar en la etimología, ámbito, diferencias y alcances de los términos citados precedentemente.

En consecuencia, nuestra preocupación se centrará en la gestión de personas, en el plano de la creación de valor organizacional y en el contexto de la armonía entre objetivos organizacionales, evaluación de desempeño y recompensa. Para estos efectos, el vocablo estratégico tendrá dos alcances fundamentales: el primero, se refiere a propósitos de largo plazo y, el segundo, a los factores y circunstancias que pueden crear o destruir patrimonio organizacional. Y, por otra parte, el foco está orientado a la concepción de una noción métrica para evaluar el desempeño y, como consecuencia, la recompensa asociada a los diferentes niveles de evaluación de desempeño medido.

¿Por qué le asignamos tal relevancia a la relación desempeño-creación de valor? La respuesta emana prácticamente espontánea a partir de las ideas presentadas en los párrafos precedentes. El constructo humano, como fenómeno general, responde al ordenamiento orientado al bien común sustentable. Así entonces, el valor organizacional creado, expresado en términos agregados, es el valor social creado, y éste debe impulsar el desarrollo humano de la sociedad como el mayor de los conglomerados o agrupación de individuos.

Entonces, la gestión de personas en la organización es uno de los ladrillos que se sumará al bienestar general del orden humano. Se infiere, entonces, que la gestión de la organización individual forma parte de la gran retícula de la sociedad. En otras palabras, cuando la gestión individual de la empresa es exitosa ésta aporta valor a la construcción de la sociedad en su conjunto. Parafraseando a Rousseau, esta suerte de Contrato Social sobre el que opera la organización, no sólo está concebido para beneficiar a los stakeholders más evidentes, sino que es verdaderamente un agente de cambio, con dinámica propia y buscando una perfecta sincronía con su ambiente.

Desde otra perspectiva, el desempeño superior del ocupante de un cargo, cuyo contenido, objetivos y funciones están alineados con la estrategia de la empresa, crea valor para ésta; y una adecuada relación entre compensación y rendimiento genera escenarios propicios para el logro de excedentes empresariales y, adicionalmente, es un factor clave para el establecimiento de un clima organizacional de orden superior. 
Este trabajo es la primera fase de una línea de investigación, que busca proponer un Modelo de Estructuramiento de Estrategia Corporativa y Capital Humano, en este caso abordando alineamiento y compensación variable.

\section{Alineando estrategia y contenido de cargos}

Al formular estrategias organizacionales uno de los aspectos centrales es la formulación de objetivos y metas $^{2}$, en íntima relación con la declaración de la Visión institucional. Asimismo, el rol y el ejercicio propio del quehacer empresarial quedan condensados en la definición de su Misión.

Lo señalado gatilla la revisión y reformulación del cuadro organizacional de la entidad, por cuanto la verticalidad (jerarquía, autoridad y dependencia) y la horizontalidad (división del trabajo), deben diseñarse de modo tal que el organigrama también esté alineado a la estrategia corporativa. La concepción de esto se observa en el Cuadro $\mathrm{N}^{\mathrm{o}} 1$, en él se aprecia la secuencia a seguir, desde las definiciones estratégicas hasta la evaluación de desempeño y compensación.

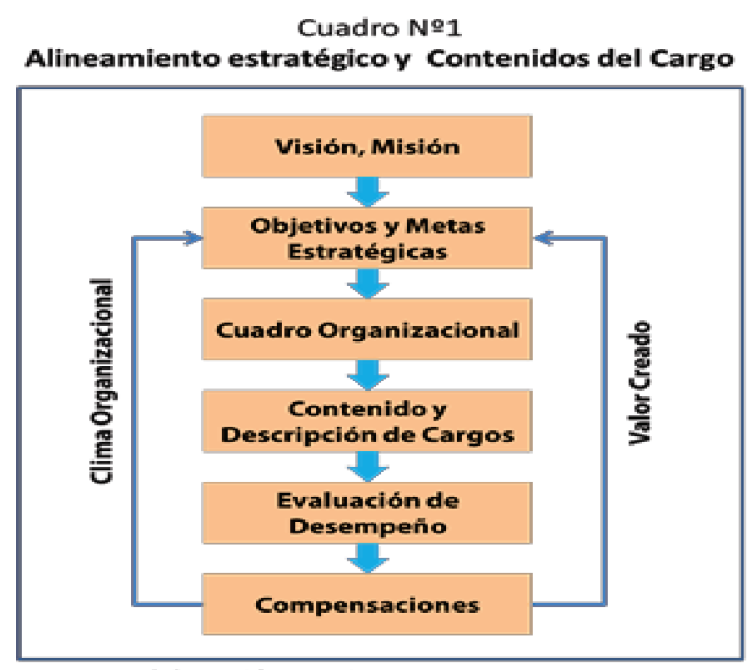

Fuente: Elaboración Propia.

Alineación de la estrategia al capital humano implica colinealidad de propósitos, es decir, si la empresa declara su Visión y Misión, es apropiado también declarar visiones y misiones en las unidades de departamentalización, con esto se consigue que cada unidad trabaje con una brújula propia en función del curso de navegación institucional. Veamos el siguiente ejemplo: La Junta Nacional de Cuerpos de Bomberos (JNCB) redefinió su Visión (BRAVO Y MIRANDA, 2009a) como "Ser reconocida por los Cuerpos de Bomberos de

\footnotetext{
2 Para efectos de este trabajo entenderemos a las metas como "objetivos cuantificados". Precisión de suma relevancia, pues cuantificar es un paso necesario para establecer métricas formales.
} 
Chile como un organismo de servicio de alta calidad en la asignación de recursos, apoyo técnico y formación integral de bomberos. Asimismo, aspira a ser el referente de la actividad de Bomberos de Chile ante el estado y la comunidad nacional e internacional". Al mismo tiempo definió la Visión para sus unidades departamentales (Organigrama), veamos los casos de la Dirección Ejecutiva: "Transformar a la JNCB en una organización que, con una fuerte vocación de servicio, administre con la mayor probidad y eficiencia los recursos que opera para el cumplimiento de los objetivos institucionales" y de la Subdirección de Finanzas: "Ser reconocida por su solidez técnica, probidad, transparencia, compromiso con el control y por el uso de los recursos en estricta correlación con las políticas superiores de JNCB".

Como se colige del ejemplo, las máximas declaraciones estratégicas de la organización, plasmadas en la Visión y Misión, se desagregaron en declaraciones análogas, y supeditadas a las institucionales, en las unidades departamentalizadas que operan cada ámbito de la JNCB. Asimismo, la institución definió (BRAVO Y MIRANDA, 2009b) sus objetivos, metas, estrategias, programas, responsables, plazos y medidas de evaluación (KPI) para darle cauce material a las declaraciones, así también cada unidad organizacional queda en condiciones de definir sus propios parámetros análogos. Es decir, en el caso, cada unidad de responsabilidad adopta su específico curso de navegación alineado al curso institucional.

Respecto de contenido de cargos, tomemos el ejemplo de la Subdirección de Finanzas del caso en comento. El primer factor declarado es la "solidez técnica", en consecuencia, serán elementos clave de quien ejerza tal función aspectos como el dominio de finanzas de largo y corto plazo, análisis financiero, gestión de tesorería, legislación aplicable como de mercado de valores, laboral e impositivo. El segundo factor es la "probidad", esto pone el acento en algunos requisitos de ingreso, pero por sobre todo en una sólida ética profesional durante el ejercicio del cargo. El tercer factor es la "transparencia", esto se centra en la continua y permanente existencia de una accountability a disposición de stakeholders externos e internos, lo que obliga a la mantención actualizada de reportes y registros conforme a la normativa y prácticas profesionales en curso. El cuarto factor es el "compromiso con el control", refiriéndose al amplio alcance de la expresión, tales como verificaciones y pruebas permanentes, análisis de desviaciones y brechas, medidas correctivas y su adecuada y oportuna exposición. El quinto factor "uso de los recursos en estricta correlación con las políticas superiores..."da cuenta de la subrogación a las declaraciones institucionales y define, entonces, los márgenes de autonomía y dependencia de la posición y se relaciona directamente con lo declarado por la JNCB en la Visión como “...organismo de servicio de alta calidad en la asignación de recursos...". 
El caso ilustra cómo se diseña y materializa el alineamiento entre estrategia y contenido de cargos, y expresa con meridiana claridad la metodología a seguir por directivos, gerentes y consultores en las organizaciones.

\section{Evaluación de desempeño}

En esta materia se dispone de un inventario de métodos, conocidos en su mayoría por directores y gerentes de recursos humanos. Dessler, G. y Varela, R. (2011) mencionan: Método de Escala de Puntuación Gráfica, Método de Clasificación Alterna, Método de Comparación con Pares, Método de Distribución Forzada, Método del Incidente Crítico, Escalas de Clasificación Basadas en el Comportamiento. Chiavenato, I. (2011) también considera algunos tales como el Método de Investigación de Campo y el Método de Frases Descriptivas. Estos y otros contienen, en mayor o menor medida, un elemento que complica su aceptación por muchos gerentes: la constante presencia de la subjetividad y la dificultad para que esta sea acotada. Este último aspecto abre un escenario de análisis e investigación, tras la búsqueda de nuevas proposiciones orientadas a medir el grado de cumplimiento de las principales actividades de un cargo, hecho de suma importancia a la luz de los antecedentes precedentemente expuestos, por cuanto constituyen el insumo para medir down-top el grado de cumplimiento a los propósitos estratégicos de la empresa.

Una dura tarea es evaluar el desempeño teniendo como referencia la cuantificación de fenómenos cualitativos. Es decir, cómo es posible medir actos vinculados a la conducta, en particular en el ambiente laboral. Ante esto, nos vemos en la obligación de recurrir al apoyo de herramientas matemáticas y estadísticas, para articular algún tipo de cimiento sobre el que construir una proposición.

\section{Descripción de cargo y funciones operacionales nucleares}

Son variados los continentes que pueden expresar lo que se conoce como Descriptor de Cargo, hay formatos básicos y complejos, transversales y específicos, también existen los desarrollados expresamente por la organización, considerando su realidad y circunstancias. Pero en todos ellos merece un acápite singular lo relativo a las actividades principales del cargo, que en adelante denominaremos "Funciones Operacionales Nucleares (FON)". Para efectos de la proposición esta nomenclatura tiene un sentido preciso, hecho que permite comprender sus características y alcance. Una dura tarea es evaluar el desempeño teniendo como referencia la cuantificación de fenómenos cualitativos. 
Ilustraremos el núcleo o razón de ser fundamental de un cargo, en el ordenamiento organizacional, con la expresión Core Position; con esto intentamos identificar la esencia del cargo, su columna vertebral, en el concierto empresa. Para satisfacer esta noción es esencial, conforme a lo señalado, establecer, tal como en el caso presentado (JNCB), cuál es la naturaleza de la posición, mediante la desagregación de la Visión de la unidad departamental.

Una vez hecho lo anterior, es decir, identificado el Core Position, entonces, el paso siguiente es la enunciación de las Funciones Operacionales Nucleares (FON), estas deben cumplir ciertos requisitos formales para ser construidas, los que se observan en el Cuadro $\mathrm{N}^{\circ}$ 2.

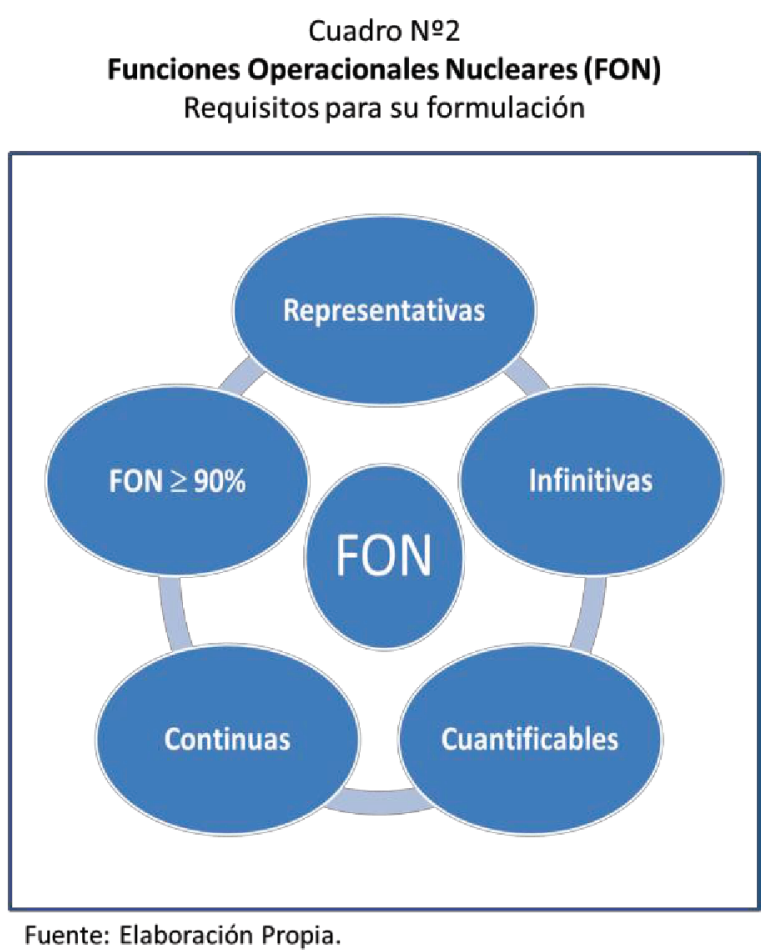

Un primer requisito a satisfacer es que una FON debe ser representativa a plenitud del Core Position del cargo, esto implica una total identificación con su rol de aportante de valor a la organización. Segundo requisito, exige que su formulación (redacción) debe ser en infinitivo, esto obliga a representar una FON como un verbo, esto es, indica una acción, concreta y diferenciable con total claridad de otra. Tercer requisito, toda FON debe ser cuantificable y susceptible de ser representada en una escala estandarizada y de comprensión general, una escala centesimal $(0 \%-100 \%)$ funciona perfectamente, pues permite comparar, ponderar y mezclar. Cuarto requisito, su propiedad "cuantificable" (tercer requisito), debe permitir que una FON pueda representarse como una función matemática continua. Quinto requisito, conforme veremos, los ensayos realizados nos aconsejan que las FON de un cargo 
no excedan de cinco (5) y que desde el prisma de la organización éstas acumulen al menos el 90\% del Core Position del cargo, debidamente ponderadas y presentadas en el descriptor del cargo en estricto orden de prelación $\left(\mathrm{FON}_{1}>\mathrm{FON}_{2}>\mathrm{FON}_{3}>\mathrm{FON} 4>\mathrm{FON}_{5}\right)$; así, en caso de existir otras funciones del cargo (Funciones Operacionales Orbitales, FO2) estas deben acumular el 10\% restante. Desde un punto de vista gerencial monitorear hasta el noveno $\left(9^{\circ}\right)$ decil representa una robusta herramienta de gestión. Es decir, la acumulación de las FON será:

$\sum \mathrm{FON}_{\mathrm{j}} \geq 90 \%$ donde $1 \leq \mathrm{j} \leq 5$

Cada FON se evaluará con su propia "variable de evaluación” o variable independiente $\left(\mathrm{v}_{\mathrm{j}}\right)$, la que alcanzará valores característicos de la FON producto de las acciones o transacciones del ocupante del cargo, y será evaluada como variable dependiente en escala centesimal (0\%-100\%), como medida de cumplimiento o de "desempeño" (EDI) para la FON en particular, esto es:

$$
\operatorname{EDI}_{\mathrm{FONj}}=\mathrm{f}\left(\mathrm{v}_{\mathrm{j}}\right)
$$

Donde "f" representa la expresión funcional y EDI la "evaluación de desempeño" para la $\mathrm{FON}_{\mathrm{j}}$. Al combinar las FON obtenemos la "evaluación de desempeño conjunta" (EDC), esto es la calidad global en el cumplimiento del cargo:

$$
\mathrm{EDC}=\Sigma \mathrm{EDI}_{\mathrm{FONj}} \mathrm{x} \mathrm{p}_{\mathrm{j}} \text { con } 1 \leq \mathrm{j} \leq 5
$$

\section{Política de evaluación de desempeño}

Aquí la proposición introduce el concepto de "Política de Evaluación de Desempeño" (PED), que representa el consenso de la Administración respecto de la forma de medir el grado de cumplimiento para los distintos cargos y sus respectivas FON. Este concepto constituye una respuesta a la aspiración directiva de eliminar o reducir la subjetividad en la evaluación de desempeño, la que queda acotada hasta la definición y formulación organizacional de la PED, de este modo las "fuentes" de subjetividad de la proposición claramente identificadas son:

1. La definición de las FON del cargo;

2. La ponderación (pj) asignada a cada FON; y

3. La definición funcional de la PED.

Una vez que la empresa ha definido completamente su política, de allí en adelante, los resultados de la evaluación de desempeño no pueden sino adoptar uno y sólo un resultado a determinados niveles de cumplimiento de las FON de cada cargo, y pueden ser convertidos en 
"aplicaciones" regulares en los sistemas de información, generando indicadores y reportes de una amplia gama. Es decir, los resultados obtenidos son comparables homogéneamente y operan como un índice de evolución; característica de gran relevancia, ya que permite entregar feedback (para la mejora y positivo), efectuar rotaciones de cargos, promociones, necesidades de capacitación y decidir desvinculaciones.

\section{Representación funcional}

La expresión concreta de una PED queda plasmada en una expresión gráfica y en un conjunto de funciones y ecuaciones.

Para ilustrar esta última fase, veamos el siguiente caso: Una empresa desea evaluar el desempeño de su Subgerente de Finanzas. El cargo, según la empresa, contribuye al alineamiento con las siguientes actividades, que ha definido como FON del cargo, situación que se observa en el Cuadro $\mathrm{N}^{\circ} 3$ :

\section{Cuadro №3}

Subgerente de Finanzas

Definición de la empresa de sus FON

$\Sigma F O N j \geq 90 \%$

\begin{tabular}{|c|l|c|}
\hline FON & \multicolumn{1}{|c|}{ DESCRIPCIÓN } & PONDERACIÓN \\
\hline FON $_{1}$ & $\begin{array}{l}\text { Mantener ciclo de caja en la } \\
\text { condición (Período medio de } \\
\text { cuentas por cobrar) } \leq \text { (Período } \\
\text { medio de cuentas por pagar) }\end{array}$ & $30 \%$ \\
\hline FON $_{2}$ & $\begin{array}{l}\text { Contratar deuda de corto plazo } \\
\text { del sistema financiero con tasas } \\
\text { (t) que cumplan t } \leq \text { t media de } \\
\text { mercado para operaciones } \\
\text { similares de los seis (6) últimos } \\
\text { meses }\end{array}$ & $25 \%$ \\
\hline FON & $\begin{array}{l}\text { Pagar a los Proveedores Clave } \\
\text { (definidos por la empresa) con } \\
\text { un máximo de 15 días de } \\
\text { retardo respecto de la fecha } \\
\text { nominal de facturas }\end{array}$ & $20 \%$ \\
\hline FON & & \\
\hline FON & & \\
\hline & D5... & $10 \%$ \\
\hline
\end{tabular}

Fuente: Elaboración Propia.

Para ilustrar esta tarea tomemos la $\mathrm{FON}_{1}$ del caso y veamos cómo la empresa ha definido evaluar el desempeño del ocupante del cargo. Esta función da cuenta del calce (o descalce) de la tesorería. Asumamos que para la empresa es relevante disponer de un calce o días a favor siempre y cuando éste sea mayor o igual a 40 días, en este negocio, a nivel de industria, es extremadamente difícil obtener calces por sobre los 60 días. Se reúnen las 
gerencias y después de un acalorado debate llegan a un acuerdo, éste es informado al Comité Ejecutivo del Directorio, facultado para aprobar o rechazar. Finalmente sale humo blanco y se aprueba la propuesta de evaluación de desempeño para la $\mathrm{FON}_{1}$ (esto que planteamos constituye una simplificación de la realidad, pues llegado el caso se presenta la PED para todas las posiciones de la empresa). La medida aprobada, en forma gráfica, queda expresada así:

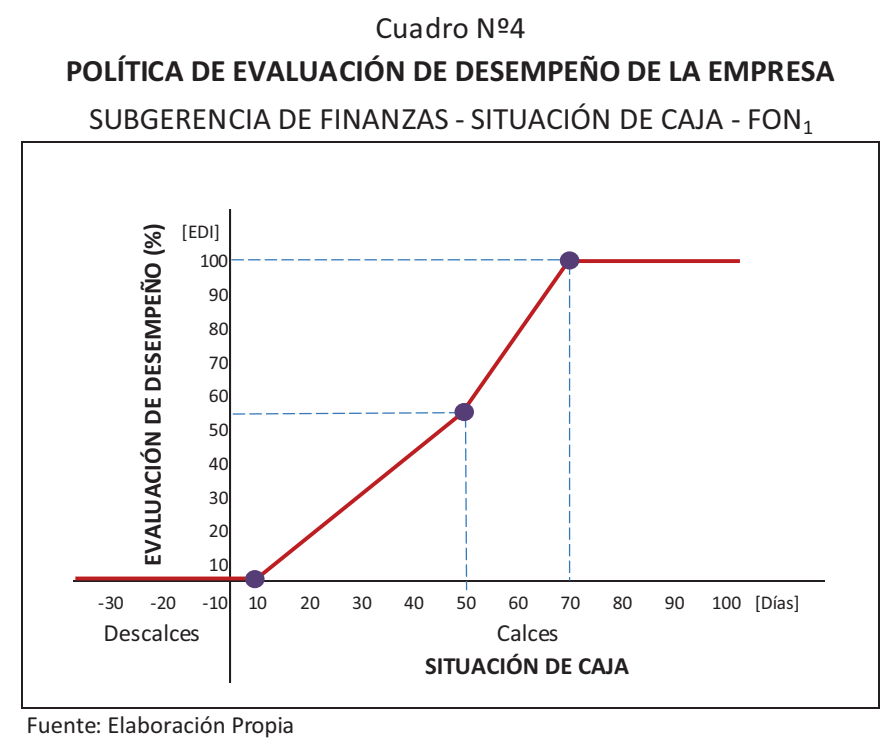

Una primera observación, para la construcción de la política, es que se propone trabajar con segmentos lineales; por dos razones, la primera, evita la formulación de polinomios y, la segunda, las funciones lineales son de cálculo rápido y de programación sencilla.

El número de funciones dependerá de los intervalos que definan la política y la valoración (eje de ordenadas) que se quiera dar a los diferentes niveles de logro de la variable de evaluación (eje de abscisas).

En forma analítica, la política se expresa:
$\mathrm{EDI}=0$
$\mathrm{Si} \quad \mathrm{D} \leq 10$
$\mathrm{EDI}=(55 / 40) \times \mathrm{D}-(55 / 4)$
Si $\quad 10<\mathrm{D} \leq 50$
$\mathrm{EDI}=(45 / 20) \times \mathrm{D}-(115 / 2)$
Si $\quad 50<\mathrm{D} \leq 70$
$\mathrm{EDI}=100$
Si $\quad \mathrm{D}>70$

Nótese que la pendiente de la función lineal correspondiente al intervalo [50;70] es mayor que la pendiente la función del intervalo $[10 ; 50]$, de modo que un incremento 
marginal de la variable evaluadora en éste último intervalo es mayor que si se produce en aquel. En otras palabras, se premia a tasas mayores el crecimiento unitario a partir de $\mathrm{D}=50$, lo que constituye un claro incentivo para el ocupante del cargo.

\section{CONCLUSIONES}

La Evaluación de Desempeño y los Descriptores de Cargo mediante la Metodología "EDAE Corporate $@$ ” representan un claro avance en el sentido de reducir y controlar la subjetividad en la medición de los resultados de los ocupantes de cargos en la organización.

Como se aprecia, a lo largo de la proposición, EDAE Corporate $₫$ es una herramienta al servicio de los propósitos estratégicos de la empresa, toda vez que se aplique la metodología a plenitud. Implementaciones parciales podrían producir sesgos y complicar aún más la situación organizacional en materia de gestión de personas.

EDAE Corporate $₫$ también es la puerta de entrada hacia el otorgamiento y la determinación de compensaciones variables con fuertes niveles de correlación con las expectativas de la empresa (resultados) respecto de la gestión de cada una de las posiciones.

El hecho de acotar los grados de subjetividad en la evaluación de desempeño, hasta la puesta en vigor de la política, representa un avance sustantivo, pues a partir del momento de su aplicación los resultados son independientes de quien efectúe la evaluación.

La Metodología EDAE Corporate $₫$ es aplicable a todo tipo de puestos, generadores o no de ingresos, de implícita naturaleza cuantitativa o no; la herramienta siempre ofrece una oportunidad de cuantificar aspectos cualitativos.

Asimismo, es aplicable en diversos sectores de la actividad económica, del mismo modo que se puede emplear en entidades públicas como en organizaciones sin fines de lucro.

Aún con estas apreciaciones, EDAE Corporate $@$ es una proposición que resuelve sólo una fracción de la problemática de gestión de personas. Resta mucho que avanzar en este campo. Mientras más sofisticadas sean nuestras actividades tendremos que enfrentar nuevos retos en materia de recursos humanos.

\section{REFERENCIAS}

BRAVO, P.; MIRANDA, J. JNCB, Plan Estratégico Institucional, Estructura General. B\&M Servicios Corporativos, Documento de Trabajo. Santiago. 2009a. 
BRAVO, P.; MIRANDA, J. JNCB, Implementación de Planificación Estratégica Institucional, Fase II, Diagnóstico Organizacional. B\&M Servicios Corporativos, Documento de Trabajo. Santiago. 2009b.

CHIAVENATO, I. Administración de Recursos Humanos. El capital humano en las organizaciones. McGraw-Hill. 9ª Edición. México. 2011.

DAVID, F. Conceptos de Administración Estratégica. Pearson Educación. $11^{\mathrm{a}}$ Edición. México. 2008.

DE LA CORTE, J.; MIRANDA, J. Declaración de Panamá Sobre Responsabilidad Social, Oportunidades y Desafíos. De La Corte \& Miranda. Santiago-Sevilla. 2012.

DESSLER, G.; VARELA, R. Administración de Recursos Humanos. Pearson Educación. $5^{\text {a }}$ Edición. México. 2011.

FRANCÉS, A. Estrategia y Planes Para la Empresa Con el Cuadro de Mando Integral. Pearson Educación. 1 ${ }^{\mathrm{a}}$ Edición. México. 2006.

GARCÍA, L.; GARCÍA, J. Impacto de la Inversión en Capital Humano Sobre el Valor Empresarial. Revista Latinoamericana de Administración. No 51, pp. 15-26. 2012.

HODGE, B. J. et al. Teoría de la Organización. Un Enfoque Estratégico. Pearson Educación.

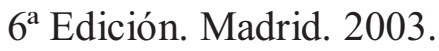

MIRANDA, J. Gestión de Personas. Herramientas Cuantitativas Aplicadas a Compensaciones. Revista Científica "Teoría, Enfoques y Aplicaciones en las Ciencias Sociales", No 5, pp. 107-116. 2010.

MONDY, R. Administración de Recursos Humanos. Pearson Educación. 11 a Edición. México. 2010.

STREBEL, P. et al. Gestión del Cambio. ¿Por Qué los Empleados se Resisten al Cambio? Deusto. $1^{\text {a }}$ Edición. Buenos Aires. 2004.

VALLE, R. La Gestión Estratégica de los Recursos Humanos. Pearson Educación. 2a Edición. Madrid. 2004.

WHEELEN, T.; HUNGER, J. Administración Estratégica y Politica de Negocios. Conceptos y Casos. Pearson Prentice Hall. 10 Edición. México. 2007. 